\title{
Automation of DMPS Manufacturing by using LabView \& PLC
}

\author{
F. Fareeza ${ }^{1}$, Chunchu Rambabu' ${ }^{2}$, S. Krishnaveni ${ }^{3}$, Abel Chernet Kabiso ${ }^{4}$ \\ ${ }^{1,3}$ Dr. M.G.R. Educational \& Research Institute, Chennai, India \\ ${ }^{2,4}$ Arba Minch University, Arba Minch 21, Ethiopia
}

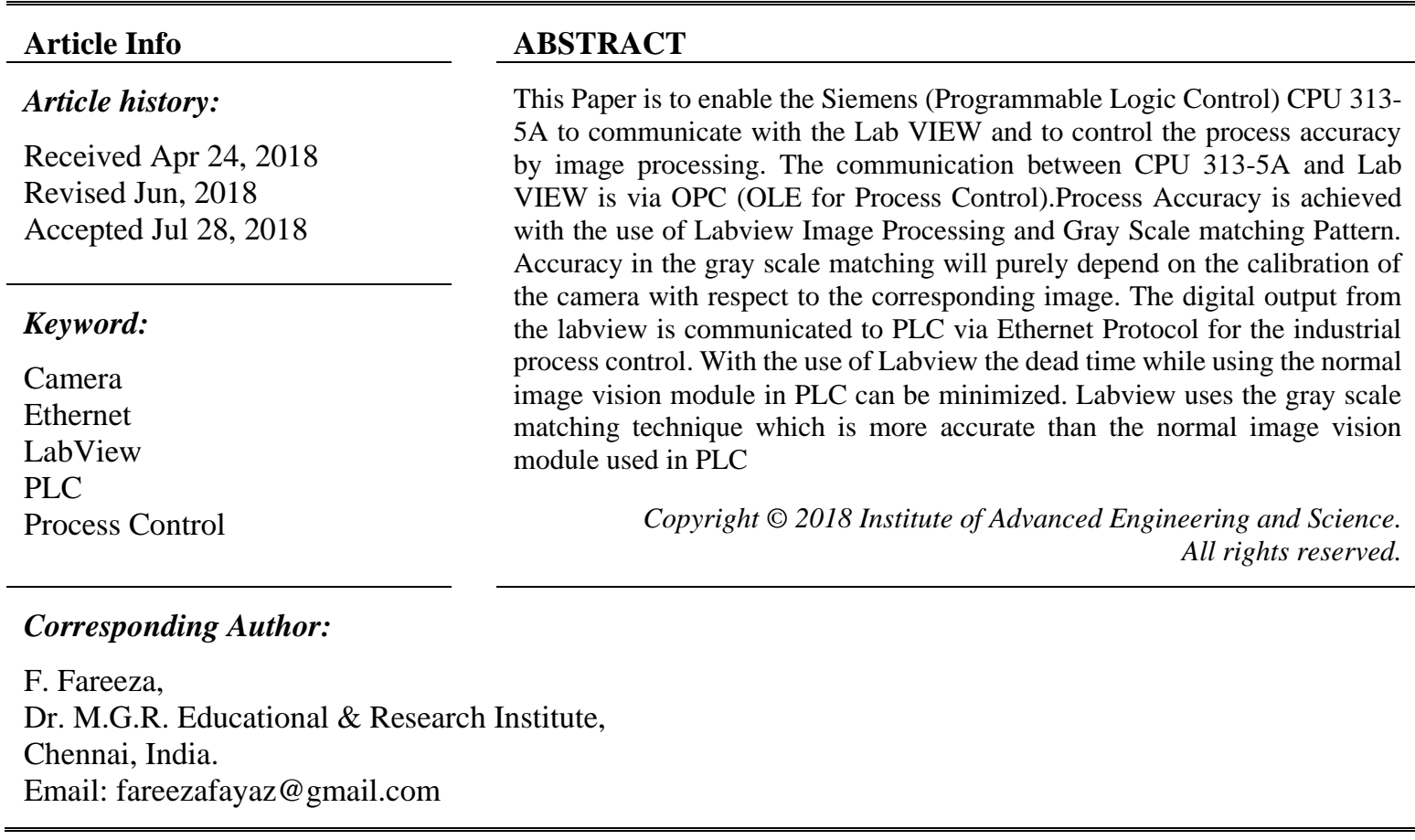

\section{INTRODUCTION}

To automate the DMPS mixing station for achieving better accuracy in the process with the use of Labview Image Acquisition module and PLC Machine vision is a broad term. Many mathematical theories, image acquisition, image processing and analysis, etc. form the whole machine vision world. Therefore, to define machine vision is a difficult task, if these things are considered. Pages and pages could be written on these different topics. For this reason, the main concentration of this thesis document was to develop a simple machine vision application using software readily available in the market. The software used in this thesis paper was Lab- VIEW. However, the basic requirements to understand a machine vision system are discussed in the forthcoming sections.

\section{LITERATURE SURVEY}

It is reported that mere physical examination and Laboratory shambling report is not helpful in the Process Accuracy, The reason behind it was the atmospheric conditions especially the temperature and the moister content may affect the Process accuracy Anjali Setal (2014), discussed about the various advantages on Labview-PLC based process control for a small statin with digital inputs via Modbus communication Protocol [1]. Forsyth et al (2009) contributed a method where measure of Image Color in gray scale pattern which is more accurate than Color matching Technique by Comparison with a reference image [2]. Papadopoulos, E et al (2008), described a novel approach to automatean industrial process by the Image matching Technique with the use of separate image acquisition module [3]. Siemens Industrial Automation, (2008) explained the operation of the CPU 315- A Series type of Programmable controllers [4]. N. N. Barsoum, et al (2011) explains remote control applications over a wide area which is commonly used in industries these days. Ethernet module was used for achieving remote control [5]. Nargalkar Akshay, et al (2005), is to enable the Allen Bradly PLC (Programmable Logic Control) SLC-500 to communicate with the 
Lab VIEW. The communication between SLC-500 and Lab VIEW is via OPC (OLE for Process Control). Development of OPC using RS Linux OPC Server. OPC is an industry standard provides real time plug-andplay software technology for process control and factory automation [6]. Fairchild, M. (2005). Proposed a new feature of Color matching Technique by Comparison with a reference image [7].

\section{DMPS}

DMPS (Dimethoxic Proponic acid) is mixed gradually under controlled temperature with Edible Oil (Sunflower) for better quality. For this process 12 Ton of Edible Oil-Sunflower is taken for a batch, in its $12 \mathrm{Kg}$ of Sunflower oil concentrated with 120 Grams of DMPS solution is mixed gradually under controlled temperature. For the preparation of DMPS concentrated Sunflower oil $12 \mathrm{~kg}$ of oil is taken in a vessel (small tank) and it was maintained at $650 \mathrm{C}$ with the use of heater coils then DMPS Solution is dosed gradually to it with the use of dosing pump. During this process the colour of the sunflower oil in the tank gets changed due the concentration of DMPS. When the Colour of the DMPS Concentrated Sunflower Oil gets changed to Lime colour the camera will sense it and sends the signals to Lab view. The command from the lab view will be communicated to PLC-Simens CPU 315-A which governs the whole process with the use of ladder logic programmed in it.

For the preparation of DMPS concentrated Sunflower oil $12 \mathrm{~kg}$ of oil is taken in a vessel (small tank) and it was maintained at $650 \mathrm{C}$ with the use of heater coils then DMPS Solution is dosed gradually to it with the use of dosing pump the control of the dosing pump, Agitator and dosing pump is governed by the PLC Omron-Cpm2a.Temperature control of the vessel is controlled locally with the use of temperature controller with signals from temperature transmitter connected with RTD-PT100.The status signal of the temperature controller is given to the PLC Omron-Cpm2a for the control of the process.

As the concentration of DMPS increases the color of the sunflower Oil changes to lime color which is sensed with the use of camera and the image is processed in labview and the status signal is shared to PLC for further action via OPC server of labview which is received by PLC via OPC client.

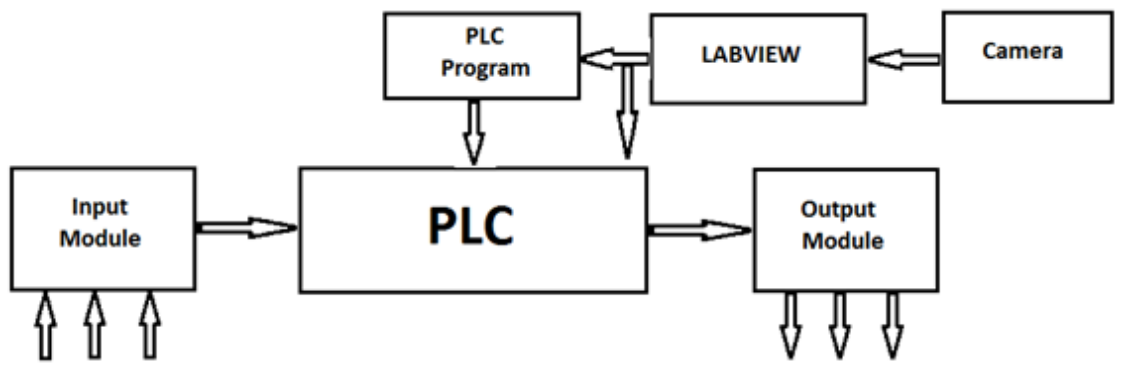

Figure 1. Block Diagram 


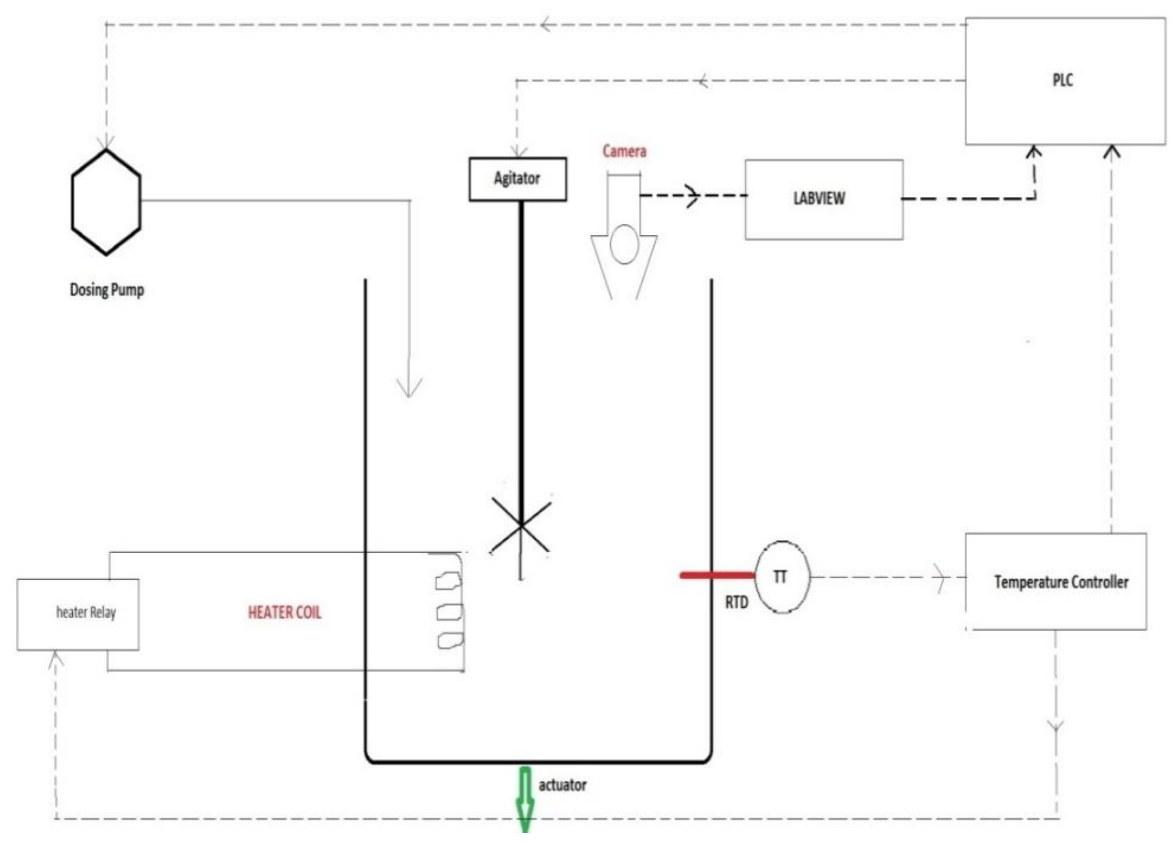

Figure 2. Process Diagram

\subsection{Process Sequence}

1. Push button ON-):Agitator, Dosing Pump and Heater gets poweredheater uses separate temperature controller loop uses temperature Controller

2. Camera Starts sensing and when it reaches the required colour it passes the signal to plc via Lab view

3. When Image and temperature satisfies condition agitator and dosing pump stops and the outlet actuator starts dropping

\subsection{Techniques Used}

1. $\quad$ PLC -Siemens CPU 315-A uses Ladder Logic Programming with

2. Step-7 Logic LabView Uses Graphical programming

3. PLC-LabView Communication is with the use of LabView OPC Server and Omron CX-One Server

\section{COMPONENTS OF MACHINE VISIONSYSTEM}

A machine vision system typically consists of machine visionsoftware (machine vision tools) and a camera (image acquisition device). Butmany otherthingsneedtobeconsideredforamachinevisionsystem.Eachof the components has its own significance. So none of the componentscan be isolated or segregated. The important components are discussed inbrief in the followingsub-sections.

\subsection{Illumination}

Illumination refers to the light sources that are available around the object being analyzed. It is significant that the object(s) under analysis be clearly visible to the image acquisition device. It ensures that much of the information is retained in the acquired image, and no much image processing needs to be done; thus making the machine vision application simpler to develop.

Illuminating object(s) does not mean availability of huge amount of light around the object; it refers the lights to be adjusted in a proper way. Proper illuminationinvolves the right intensity and correct direction of light. It should be done in a way that shadow formation is checked and maximum contrasts can be achieved from the region of interest of the object(s). (Mo- vimed custom imaging solutions, 2007.)

The light sources may be fluorescent lights or LED lights or halogen lights, etc. LED lights are more preferred over the other types of light sources, because of their long life and less energy consumption. Depending upon the arrangement of lights, illumination can be direct or indirect. 


\subsection{Imageacquisition}

Image acquisition is the most important part in a machine vision system. It involves capturing an image of the object to be analyzed with the help of camera. Different types of cameras can be used for image acquisition; they can include an ordinary mobile camera, a typical digital camera, or even a webcam. But cameras that are tailored specially for industrial use are also available. Depending on the sensor technology used, different cameras can be classified into two categories as follows:

- CMOS cameras

- $\quad$ CCD cameras

"The sensors could be matrix sensors or line sensors. An image sensor converts an optical image into an electronic signal"

Choosing a machine vision camera can be a difficult task. However, resolution, sensitivity, and type of cameramonochrome or color, should be considered when buying one. (ALLIED Vision Technologies GMBH, 2006.) Also, the interface the camera uses for communication should be considered. The available interfaces include USB, Ethernet, Firewire, etc.

\subsection{CCD Cameras}

A CCD (Charge-coupled Device) camera uses the CCD sensor technology. The main features of these sensors (and hence cameras) are listed as follows. (Vision Systems Oy. 2012.)

The most common camera sensors In-coming charges are stored Equivalent to films of traditional film cameras.

Consist of pixels with a typical size of $10 \mu \mathrm{m} \times 10 \mu \mathrm{m}$.

- These are both color and monochrome.

- These are light-sensitive diode sensors.

- Each pixel has a micro-lens for focus- ing the light into the sensor surface.

- Disadvantage of these sensors is the possibility of over exposure.

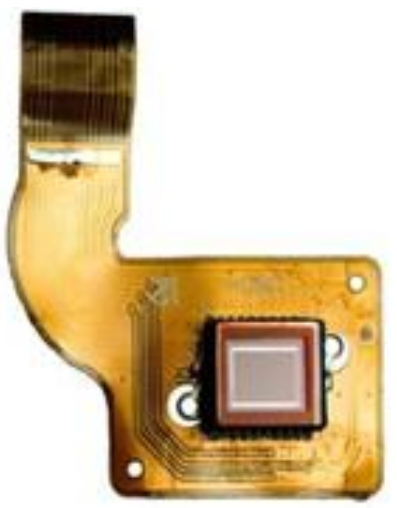

Figure 3. CCD Camera

\subsection{Depth of Field}

Depth of Field (DOF) is the measure of the distance between the nearest and the farthest objects in a scene that can be captured by the camera and be acceptably sharp in the image

\subsection{Construction of the Station}

The system consists of cylinders, sensors, a motor as the physical components. As the PLC used in the station requires additional signal modules for the $\mathrm{I} / \mathrm{O}$ (inputs and outputs) devices, signal modules from Beckhoff (www.beckhoff.com) was used.

\subsection{System Communication}

The PLC was interfaced to the computer using S7 MPI adapter. The bus coupler, LC3100 was networked with the PLC using PROFIBUS cable. Also OPC communication was done using the same S7 MPI adapter. A di- agrammatic representation of communication among the system compo- nents is shown in Figure 4. 


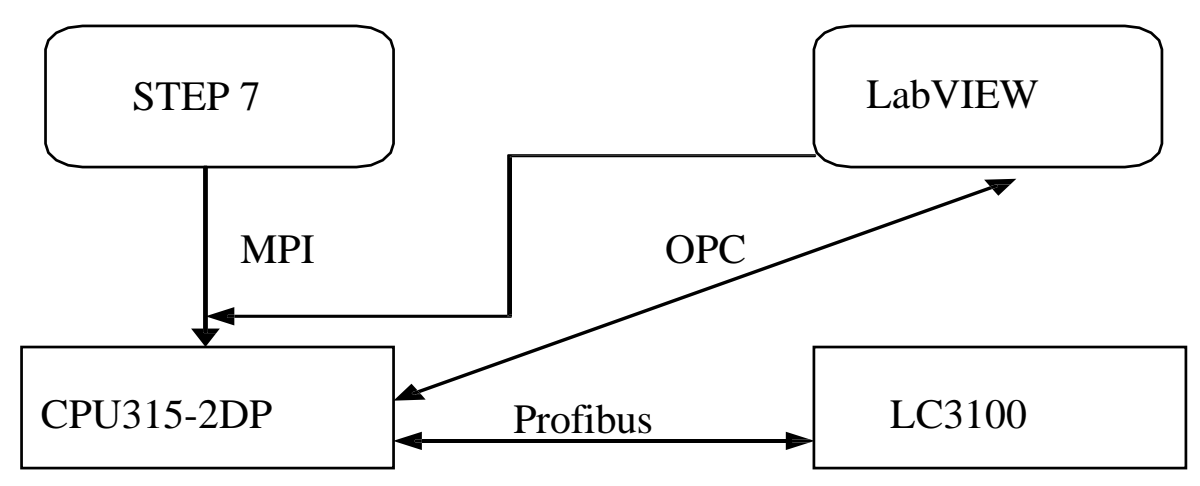

Figure 4. Communications among STEP 7, LabVIEW and CPU315-2DP and LC3100

\subsection{STEP 7 Professional}

STEP 7 PROFESSIONAL (STEP 7 in short) is automation softwarefrom Siemens Industry (www.siemens.com). It is used for program mingsimatic PLC stations. Figure 28 shows the system manger window of simatic STEP 7, where a created paper with some blocks is also shown

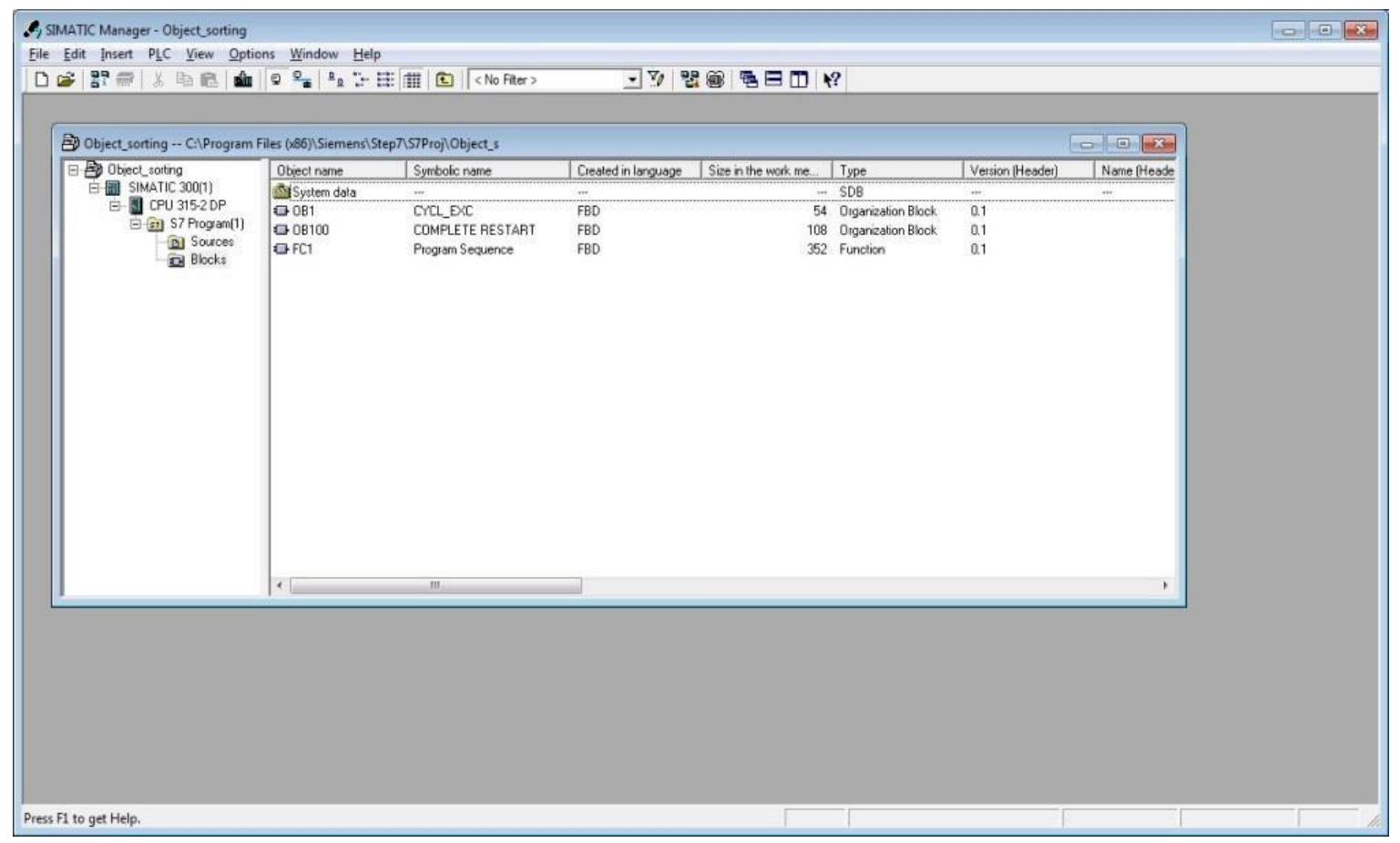

Figure 5. Screenshot of Simatic Manager (STEP 7)

STEP7 provides provision to code the program using seven differentlan-guages. Some of them are FBD (Function Block Diagram), LD (Ladder Diagram), and SCL (StatementList), etc. The user can freely choose the language. An STEP 7 auto mation program may contain functions (FCs), function blocks (FBs), organization blocks (OBs), sequence functional charts (SFCs), etc. But, every STEP 7 program must have OB1, becauseit is the main function. Details about the STEP 7 programs are not coveredin this thesis. The language used for the automation of the system described in this thesis was FBD 

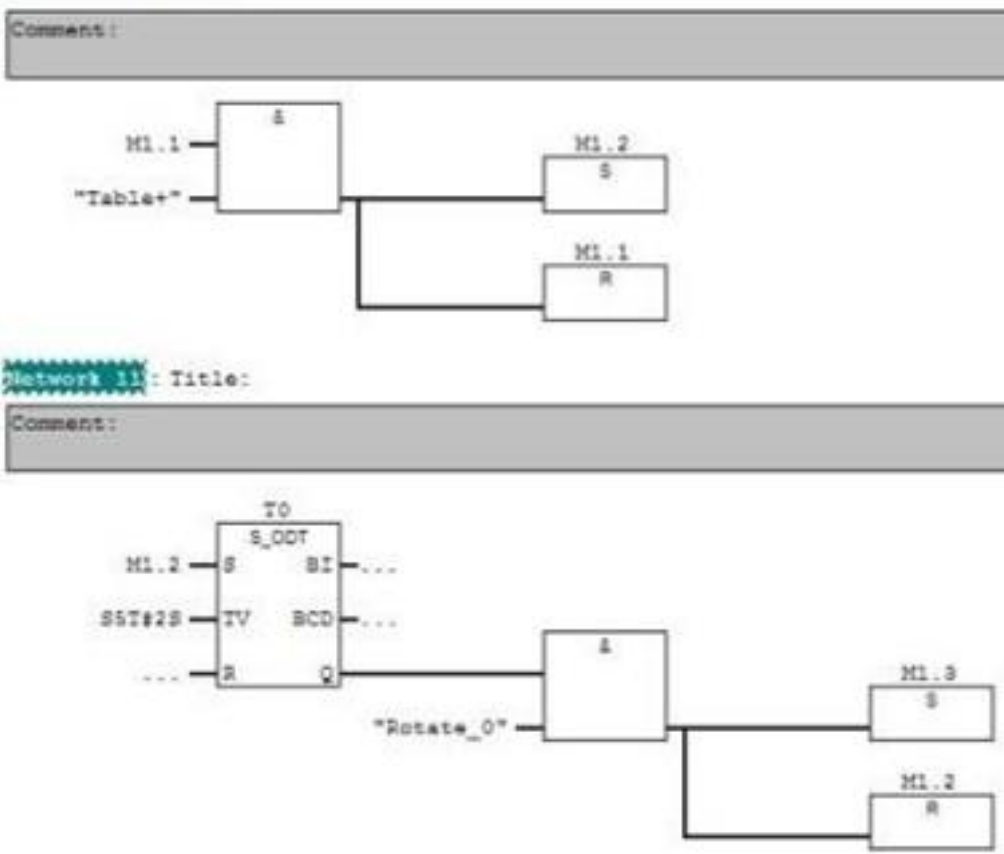

Figure 6. FBD codes in STEP 7

\subsection{CPU315-2DP}

CPU315-2DP belongs to Simatic 300 group of PLC controllers. It consists of different indicators, a program manipulation key, and a memory card slot. It has 2 serial-connection ports; one of them is for interfacing between STEP7 and the PLC whereas the other is for connecting to a distributed module. Figure 30 alongside shows CPU315- 2DP

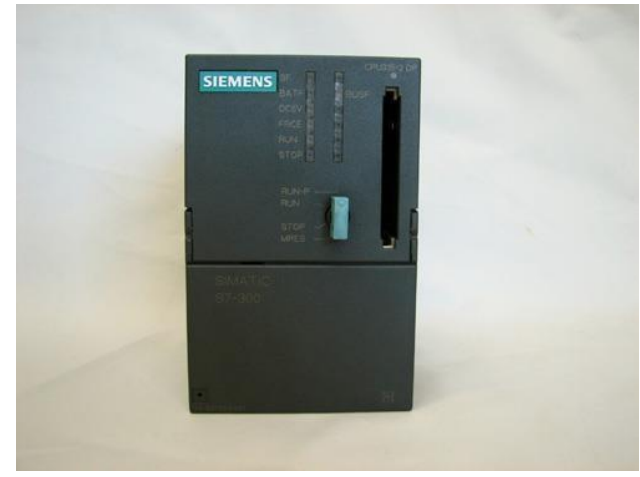

Figure 7. CPU315-2DP

\subsection{Hardware Configuration}

Hardware configuration (HW) needs to be done before any program can be downloaded into the PLC. In the hardware configuration, the type of power supply, the CPU model (such as CPU315-2DP), the signal modules and (or) other distributed modules being used need to be specified sothat the software and the hardware can inter-connect. Figure 8 shows the hardware configuration done for this paper 


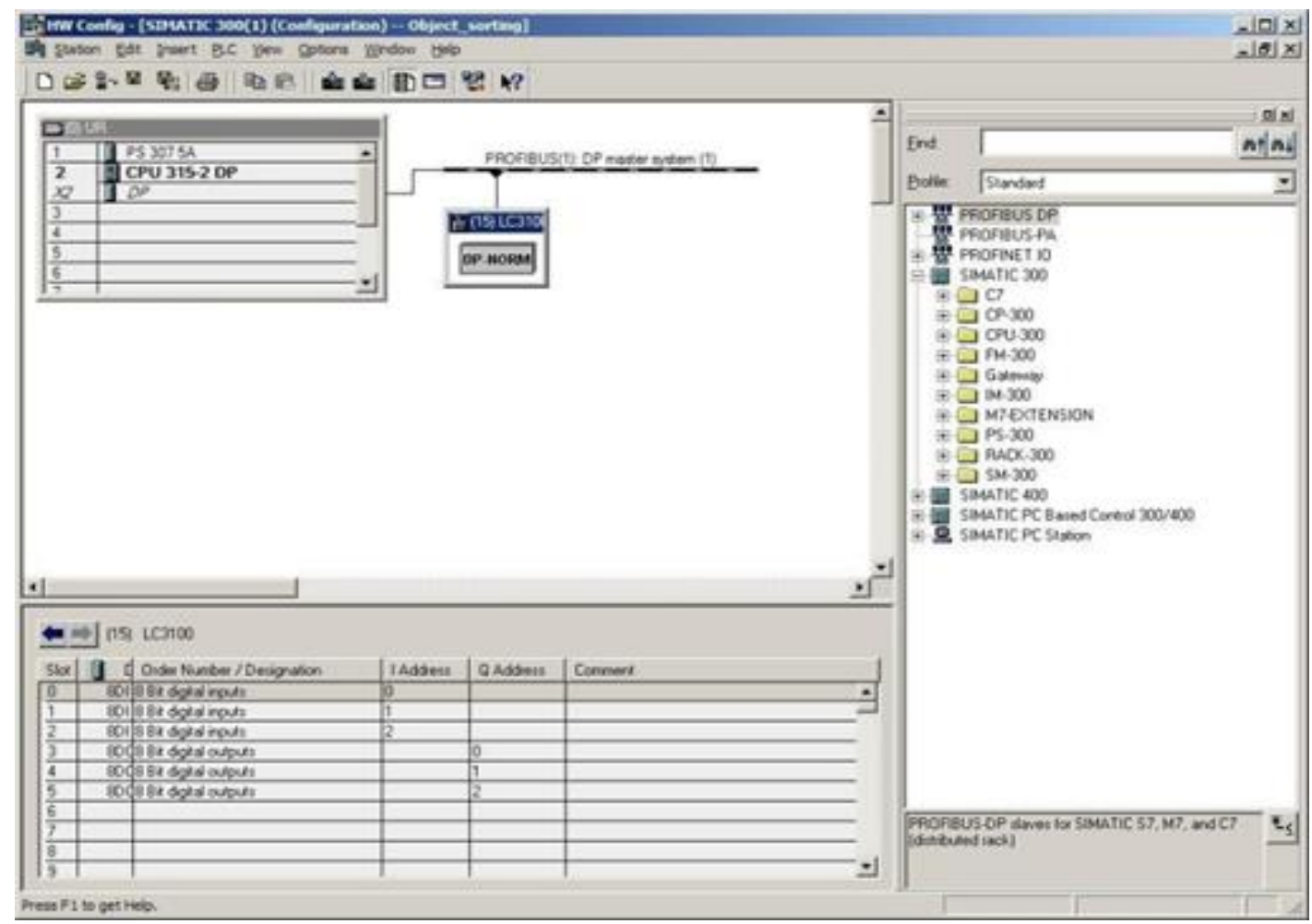

Figure 8. An example Hardware configuration for simatic PLC

\section{RESULTS AND DISCUSSION}

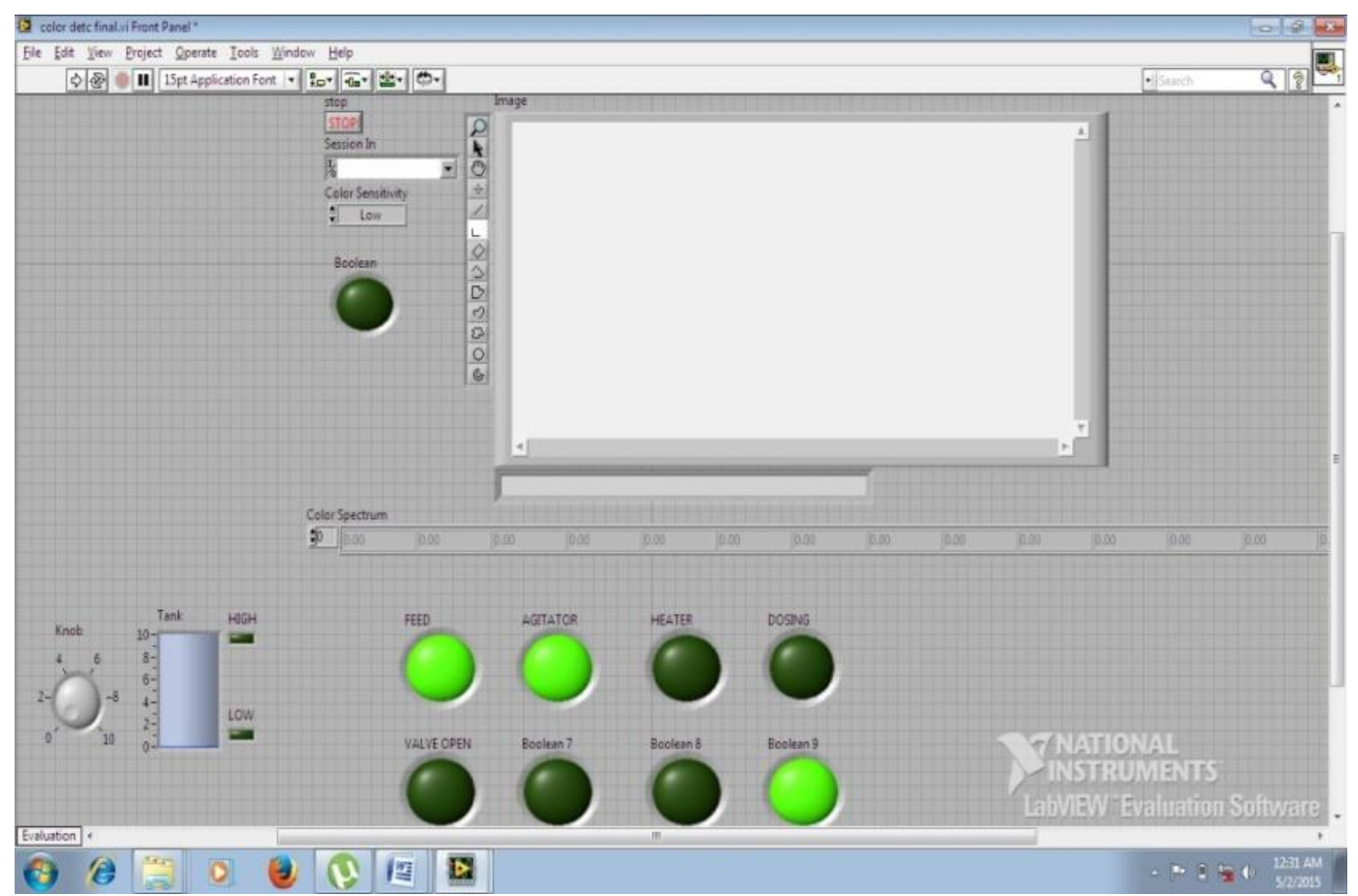

Figure 9. Labview Font Panel 


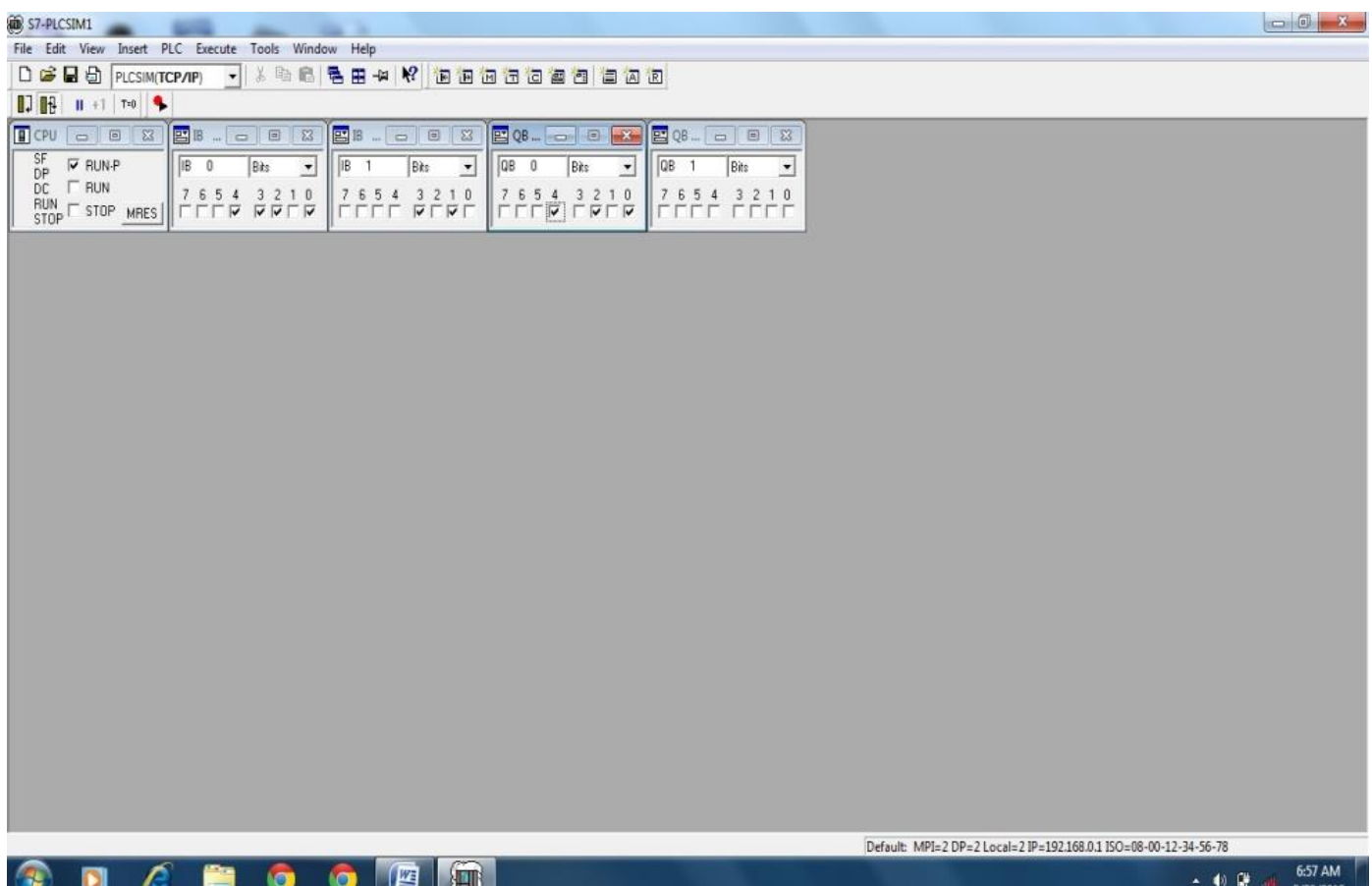

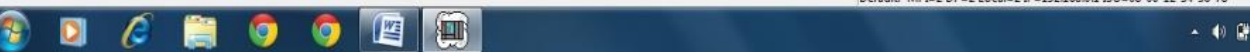

Figure 10. Step 7 Ladder and Simulation Module

The output terminal from the Unbundleby Name VI was once again wired to Array-to-Cluster VI, whose out put terminal was also once again wired to another Unbundleby Name VI. This time it was en larged to get 4 output terminals. The first output terminal was assigned to 'Bounding Box $[0]>x$ '; the second to 'Bounding Box [1]>y'; thethird to 'BoundingBox [2]>x'; and the fourth to'BoundingBox [3]>y'as shown in Figure11

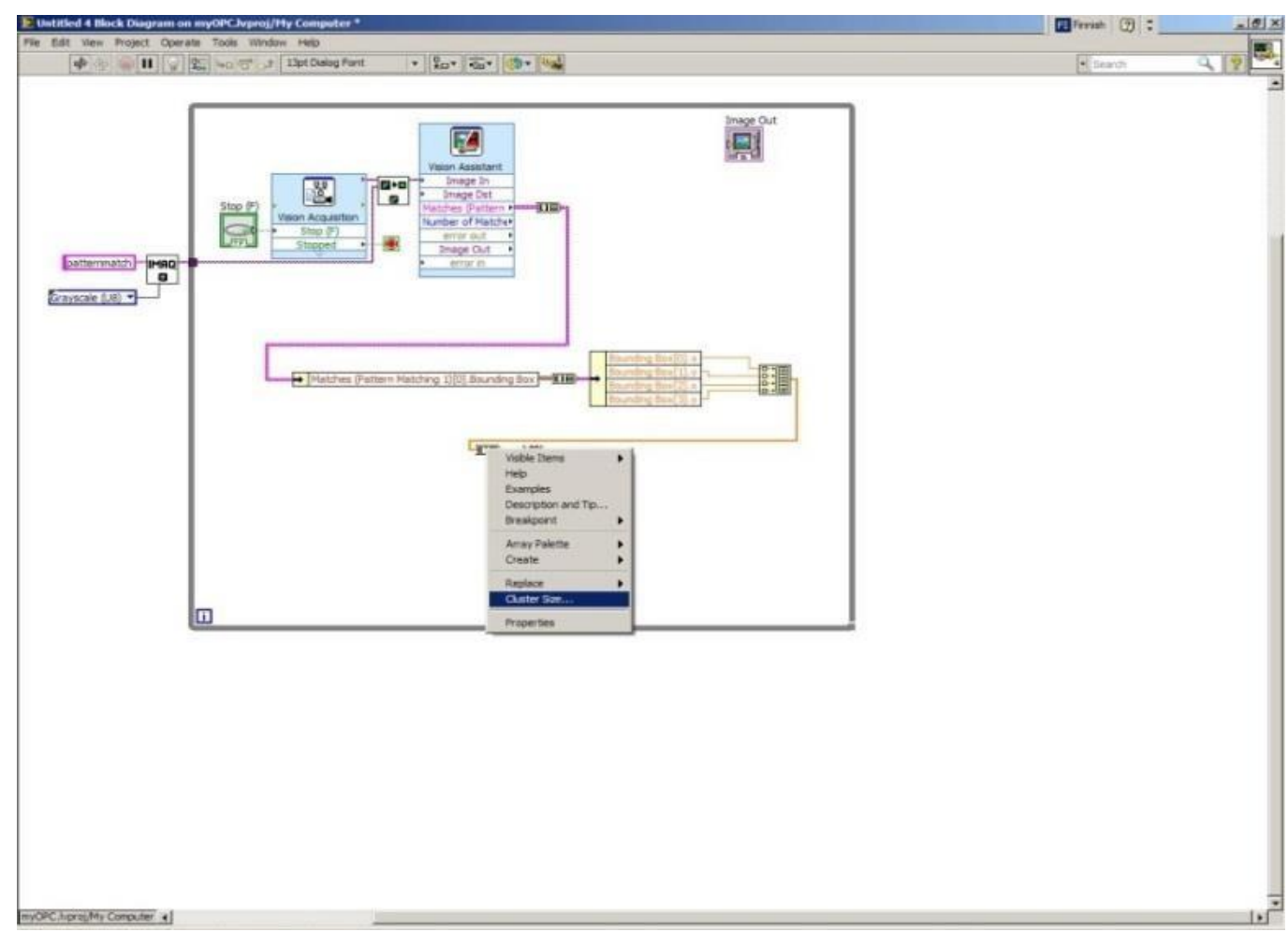

Figure 12. Changing the cluster size 


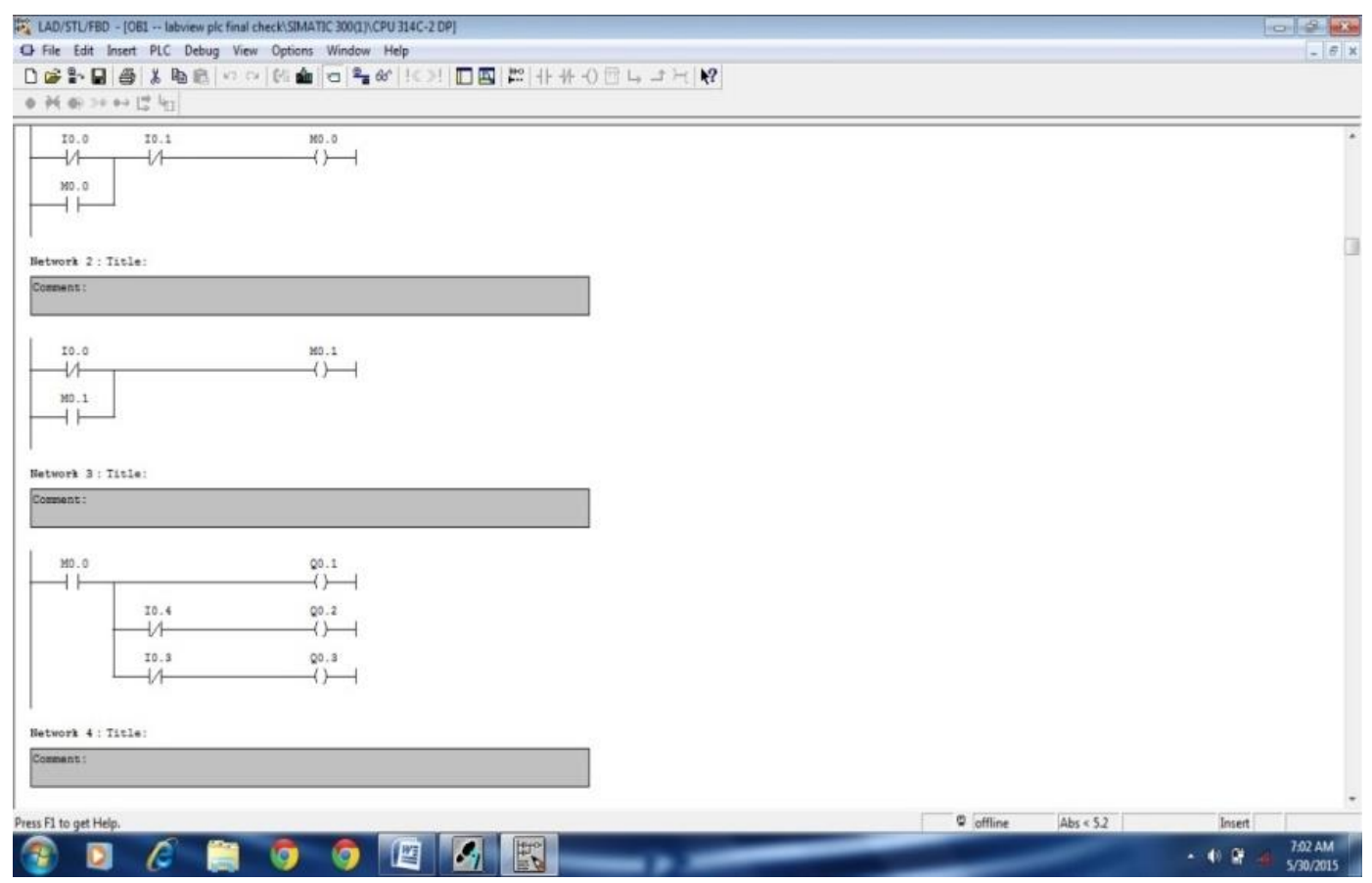

Figure 13. PLC Ladder \& Simulation Module

The output terminal from that final Array-to-Cluster VI was connected to the 'Rectangle' input terminal of the 'IMAQ Overlay Rectangle'VI. Also, the input terminal 'Image'of this VI was wired to the 'Image out' output terminal of the vision assistant. Finally, the output terminal from the Overlay Rectangle VI was wired to the input terminal of the 'Image Display'VI.

\subsection{Method of Interfacing} CONFIGURING PLC DRIVER

Here we choose RS-232 DF1 devices and click on Add New. Next we name the PLC and then start the configuration in Configure RS-232 DF1 devices.

\section{CREATING NEW OPC TOPIC}

Create a new OPC Topic and save it for further use. All the inputs and outputs that are available in the PLC will be available under topic which we have created

\section{CONCLUSION}

From the paper performed, it was obvious that machine vision technology and NI Vision tools together can be used for sorting objects in a factory production line. It was confirmed that out of many machine vision tools, the pattern-matching algorithm could be applied for the object sorting purpose. Also, it was clear that a simple webcam could be used for performing machine visions tasks. Because, a webcam was used for pattern matching, it is not for sure that it can be used for different machine vision applications. But, if the application is independent of different image processing tasks, it might be possible to use webcams instead of other industrial cameras.

The pattern matching was done at different times of the day; every time the score generated by the vision assistant needed adjustment for the correct pattern matching. This shows that pattern matching is based on direct correlation matrix algorithm. The score generated for the image template was 900 . If the score was increased over 900, none of the objects matched the pattern; below 900 pattern matched, but if the score was below certain score (for example 850), the objects which tend to be of the same pattern also matched. In that case, the aim of pattern matching failed.

Although pattern matching is not affected by lighting, it was seen that shadow formation of some other objects over the analyzed object hindered to match the pattern stored in the pattern template. So to say that pattern matching is independent of lighting might be somewhat unrealistic. It might be said that pattern 
matching not affected by lighting directly, but there might be some other indirect cause relating to light that affects pat- tern matching.

It was also confirmed that PLC could be integrated with LabVIEW using OPC communication protocol; communication was enabled using NI OPC Servers in this thesis. The integration of simatic PLC was illustrated in this thesis but almost all the PLCs can be integrated with LabVIEW using NI OPC servers; provided there is an OPC driver for the PLC.

In this thesis document it was possible to present only a simple pattern recognition technique for the object sorting. Although the objective of sorting object using machine vision tool was met, it was realized that geometric matching tool would be more robust for sorting objects. The objects used in the paper were all similar; defining only one parameter could be enough in such condition to get the result. But the case would not be the same always. The objects may have different shapes and sizes; in such cases, it is not enough to match only the pattern of the object. Measuring distances between two points, measuring diameters, etc. could be added to the application to make it more powerful and error-free.

The main idea was to interface LabVIEW and PLC for more effective and efficient process control. This paper of interfacing two most powerful technologies ruling the industries lead to many new features like acquiring data at faster rate, controlling the process accurately, providing Multi-Tasking operations, Remote controlling and several other features. Used correctly, multithreading offers numerous benefits including more efficient CPU use, better system reliability, and improved performance on multiprocessor computers. Using LabVIEW, we can start today to maximize performance on multithreaded operating systems and multiprocessor computers without increasing either your development time or the complexity of your application. Because the multithreading technology of LabVIEW is implemented transparently, no extra programming is required to take full advantage of multithreading technologies.

\section{ACKNOWLEDGEMENTS}

We, authors of this paper would like to show our gratitude to our family, friends and colleagues for sharing their pearls of wisdom with us during the course of this research paper.

\section{REFERENCES}

[1] Anjali S,et al(2014)., Application of MODBUS to Communicate the PLC and Lab VIEW for Real Time Process Control, International Journal of Emerging Science and Engineering (IJESE) ISSN: 2319 - 6378, Volume-1, Issue11, September 2013

[2] Forsyth, et al (2009) Computer Vision: A Modern Approach,

[3] Papadopoulos, E et al (2008) Pattern Analysis and Machine Intelligence

[4] Siemens Industrial Automation, (2008) "CPU 315-A Series Programmable Controller," Simens, Technique Report W393-E1-14.

[5] N. N. Barsoum, et al (2011) "Ethernet Control AC Motor via PLC Using LabVIEW, Intelligent Control and Automation, 2011, 2, 330-339.

[6] Nargalkar Akshay, et al (2005) "Real Time Automated Control of Industrial Processes with PLC -LABVIEW Communication", International Journal for Research in Science \& Advanced Technologies ISSN: 2319-2690 Issue1, Volume-1, pp 035-

[7] Fairchild, M. (2005). Color ApperanceModeles. Chichester, UK: Wiley-IS\&T proposed a new feature of Color matching Technique by Comparison with a reference image.

[8] ALLIED Vision Technologies 2006. Application Note: Choosing a Machine Vision Camera. Accessed 15th March 2013.http://www.alliedvisiontec.com/fileadmin/content/PDF/Support/Application_Notes/ALL_ApplNote_Choosin g_A_Machine_Vision_Camera.pdf

[9] Basic Photography Tutorials 2001. An Introduction to Exposure. Accessed 16th March 2013.http://www.silverlight.co.uk/tutorials/compose_expose/exposure.html

[10] Davies, E. 2012. Computer and Machine Vision. 4th edition. Waltham: Academic Press. EURESYS 2005. ADR Technology. Accessed 18th March 2013. http://www.euresys.com/Products/grablink/ADRTechnology.asp

[11] Gonzalez, R., Woods, R. \&Eddins, S. 2009. Digital Image Processing using Matlab. 2nd edition. Gatesmark Publishing.

[12] Logitech 2010. Logitech HD WebCam C615. Accessed 15th March 2013. http://www.logitech.com/enus/product/hd-webcam-c615

[13] Lode's Computer Graphics Tutorial 2004. Image Filtering. Accessed 30th April 2013. http://lodev.org/cgtutor/filtering.html

[14] Machine Vision, pdf-file (ebook). Accessed 15th January-23rd April 2013. http://www.cse.usf.edu/ r1k/MachineVisionBook/MachineVision.pdf

[15] Movimed Custom Imaging Solutions 2007. Illumination. Accessed 16th April 2013. http://www.movimed.com/Illumination.htm

[16] National Instruments 2013. Connect LabVIEW to any industrial Network and PLC. Accessed 13th January 2013. http://www.ni.com/white-paper/5407/en

[17] National Instruments 2012. Introduction to OPC. Accessed 7th March 2013. http://www.ni.com/white-paper/7451/en 
[18] National Instruments 2012. LabVIEW 2012 Datalogging and Supervisory Control Module Help. Accessed 19th March 2013. http://digital.ni.com/manuals.nsf/websearch/51AA757C42C65F0D86257A0C0029ABD0

[19] National Instruments 2008. Robotics Fundamental Series: Feature Extraction. Accessed 18th April 2013. http://www.ni.com/white-paper/8222/en

[20] National Instruments 2005. NI Vision for LabVIEW User Manual. Accessed 21st Febru- ary 2013. http://www.ni.com/pdf/manuals/371007b.pdf

[21] National Instruments 2006. Counting Particles or Cells Using IMAQ. Accessed 19th March 2013. http://www.ni.com/white-paper/316

[22] National Instruments 2011. Geometric Matching Example. Accessed 22nd February 2013. http://zone.ni.com/devzone/cda/epd/p/id/5555

[23] National Instruments 2011. Bar Code Example. Accessed 23rd March 2013. http://zone.ni.com/devzone/cda/epd/p/id/5533

[24] Pokharel, B. 2013. HAMK University of Applied Sciences. Degree Programme in Au- tomation Engineering (20092013).

[25] Quality Digest 2001. The Future of Machine Vision. Site Editor John E. Agapakis. Ac- cessed 7th January 2013 http://www.qualitydigest.com/oct $98 / \mathrm{html} / \mathrm{machfutr} . \mathrm{html}$

[26] Sudcamp 2012. A Technology Hub. Accessed 23rd March 2013. http://www.sudcamp.com/ccd-vs-cmos-imagesensor-in-digital-camera/

[27] Vision Systems Oy. 2012. Robolapaper. Education Materials.

[28] Wikimedia Commons 2012. File: Ccd-sensor.jpg. Accessed 18th March 2013. http://commons.wikimedia.org/wiki/File:Ccd-sensor.jpg

[29] WiseGeek 2011. What is Machine Vision Image Processing. Site Editor Andrew Jones. Accessed 20th January 2013.

[30] http://www.wisegeek.com/what-is-machine-vision-image-processing.htm\#did-you- know

\section{BIOGRAPHIES OF AUTHORS}
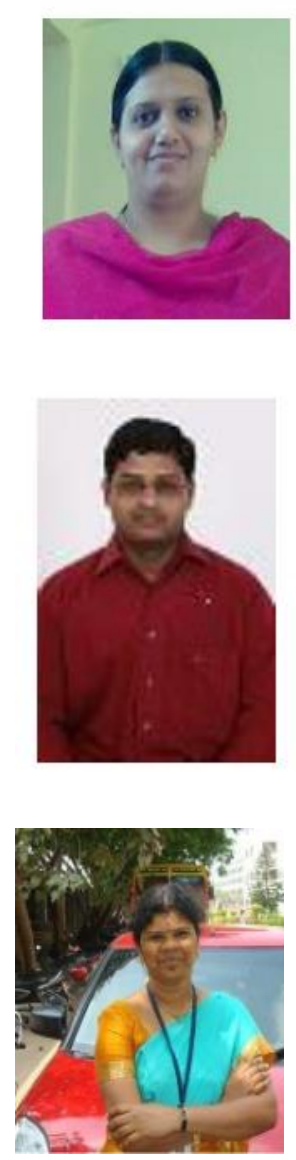

My name is Fareeza $\mathrm{F}$

I was born in 1983. I completed B.E. (Electronics \& Instrumentation) from University of Madras, , Chennai in 2004. I have completed M.Tech. (Instrumentation \& Control) from Bharath Institute of Higher Education \& Research (Deemed University), Chennai. I have completed Ph.D. (Instrumentation) from Rayalaseema Univeristy in 2018. I have a total of 11 years at Dr. M.G.R. Educational \& Research Institute, Chennai in Department of Electrical \& Electronics Engineering.

My name is Chunchu Rambabu

I was born in 1980. I completed B.Tech. (Electronics \& Control) from Jawharlal Technical University, Hyderabad in 2004. I have completed M.Tech. (Instrumentation \& Control) from Bharath Institute of Higher Education \& Research (Deemed University), Chennai. I have completed Ph.D. (Instrumentation) from Rayalaseema Univeristy in 2018. I have a total of 11 years. Currently working as Lecturer in Arba Minch University, Sawla Campus

\section{My name is S.Krishnaveni}

I had completed B.E. from the Department of Electrical and Electronics Engineering, Mepco Schlenk engineering college, Madurai Kamaraj University, Tamil Nadu, in 1996 and got my Master of Engineering degree First Class in Power Electronics and Industrial Drives, Sathyabama University, Tamil Nadu, India in June 2003. I have teaching experience of more than 19 years. For the past 13 years, Iam working as an Associate professor in EEE department in Dr M.G.R Educational and research institute university, Chennai, Tamil Nadu. I am a Professional member and advisor for 2 IEEE societies namely Industrial Application society and Women in Engineering. 


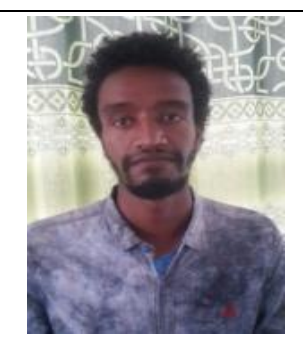

My name is Abel Chernet Kabiso

I was born form my father ChernetKabiso and mother TagesechHadero in 1994, I graduated from Addis Ababa Science and Technology University in 2017/18 BSc Electromechanical Engineering and currently I'm working as Department Head of Electromechanical Engineering in Arba Minch University Sawla Campus. 